\title{
Influence of Seed Priming Treatments on Biochemical Parameters of Dry Direct Sown Rice (Oryza sativa L.)
}

\author{
R. Pavani*, V. Umamahesh, A.R. Nirmal Kumar and Y. Reddi Ramu
}

Department of Crop Physiology, S.V. Agricultural College, Tirupati- 517502, A.P., India

*Corresponding author:

\section{A B S T R A C T}

\begin{tabular}{|l|}
\hline Ke y w or d s \\
Seed priming, \\
Gibberllic acid, \\
$\mathrm{KNO}_{3}$, Alpha- \\
amylase \\
\hline Article Info \\
\hline $\begin{array}{l}\text { Accepted: } \\
24 \text { November } 2018 \\
\text { Available Online: } \\
\text { 10 December } 2018\end{array}$ \\
\hline
\end{tabular}

The present investigation was carried out at S.V Agricultural College, Tirupati to know the effect of seed priming on biochemical parameters of dry direct sown rice (Oryza Sativa L.). A laboratory experiment was conducted in completely randomized design and replicated thrice with six popular aerobic rice genotypes i.e. MTU 1010, MTU 7029, JGL 20171, NLR33671, MTU 1075 and MTU 1112. Rice seeds were subjected to different concentrations of gibberillic acid with, 200, 500 and 1000 PPM and combination of both gibberillic acid and $\mathrm{KNO}_{3}$ treatments i.e. $\mathrm{GA}_{3}$ (200PPM, 500PPM, 1000PPM $+\mathrm{KNO}_{3}$ @3\%), $\mathrm{KNO}_{3} @ 3 \%$ and control (Hydropriming/Water soaking), in order to know the effect of seed priming (Gibberllic acid and $\mathrm{KNO}_{3}$ ) on various biochemical parameters like Reducing sugars (mg/g), Starch (mg/g) and Alpha amylase (\%) activity were recorded at 2, 4 and 6 DAT. The results revealed that in all the biochemical parameters among Varieties MTU 1010, JGL 20171, NLR 33671 had recorded higher amount of reducing sugars, starch and Alpha amylase activity compared to MTU 7029, MTU 1075 and MTU 1112. Among treatments $1000 \mathrm{PPM} \mathrm{GA}_{3}, \mathrm{GA}_{3}$ (200 PPM, 500PPM, 1000PPM+KNO $@ 3 \%$ ) are found to be best.

\section{Introduction}

Rice (Oryza sativa L.) is the most important cereal food crop of the developing world and the staple food of more than half of the world's population. Globally rice is grown over an area of 161.83 million ha with an annual production of 717.8 million tonnes (IRRI, 2017). Irrigated rice is the major consumer of fresh water. It was estimated that by 2025, about 15-20 million hectares of irrigated rice will be affected due to water scarcity which threatens the productivity. Combining the growing demand for food with increasing water scarcity, rice producers in Asia need to produce more rice with less water.

A major challenge in rice production is therefore to save water while maintaining or even increasing the grain yield (Yang and Zhang, 2010). Many water-saving technologies are currently used in rice production, including alternate wetting and drying irrigation, the rice intensification system, aerobic rice and the ground cover rice production systems (GCRPSs) (Qin et al., 2006). Among these aerobic rice is gaining 
popularity as a strategy for water saving agriculture.

Aerobic rice can achieve yields of 4-6 tons per hectare with $50-70 \%$ less water compared to lowland rice it does not require flooded wetland (Qin et al., 2010). In aerobic rice production, the seeds are direct-seeded in aerobic soil without any standing water layer, which minimizes water use and boosts up water productivity by eliminating continuous seepage and percolation, reducing evaporation and eliminating wetland preparation (Nie et al., 2012; Singh et al., 2008). Season-long weed infestation in aerobic rice may cause yield reduction up to $80 \%$ or complete failure of crop in extreme cases (Jayadeva et al., 2011; Sunil et al., 2010).

Therefore, the aerobic rice cultivars should have the capacity of early seedling establishment, quick crop growth and yield stability. Identification of strong weed competitive rice cultivar is a feasible solution to inhibit the growth of weeds and it is a costeffective and safe tool for weed management (Zhao et al., 2006).

Seedling vigour is a physiological trait and a sign of potential seed germination, seedling growth and tolerance to adverse climatic factors. On the other hand, it significantly improves the speed, uniformity and the final percentage of germination, and leads to ideal field appearance with good potential grain yield under direct-seeded conditions (Foolad et al., 2007).

Thus, to suppress weed growth, early seedling vigour of an elite variety should be achieved. Seed priming is a viable option to attain this target. Seed priming, which is also called seed hardening, is a physiological seed enhancement method. It is a presowing treatment in which seeds are soaked in an osmotic solution that allows them to imbibe water and go through the first stages of germination, but does not permit radicle protrusion through the seed coat. Subsequently, the seeds can be dried to attain their original moisture content and stored or planted using conventional techniques.

Thus in this paper the influence of seed priming with $\mathrm{GA}_{3}$ and $\mathrm{KNO}_{3}$ either alone or in combination was tested to know the amount of reducing sugars, starch and amylase content in rice seeds.

\section{Materials and Methods}

$0.1,0.25$ and $0.5 \mathrm{~g}$ each of $\mathrm{GA}_{3}$ (gibberellic acid, HIMEDIA) was dissolved separately 500 $\mathrm{ml}$ each in DDW along with $1.5 \mathrm{~g} \mathrm{KNO}_{3}$ in each case to prepare a series of solutions that gives $\mathrm{GA}_{3} @ 200 \mathrm{ppm}+\mathrm{KNO}_{3} @ 3 \%$, GA3 @ $500 \mathrm{ppm}+\mathrm{KNO}_{3} @ 3 \%, \mathrm{GA}_{3} @ 1000$ $\mathrm{ppm}+\mathrm{KNO}_{3} @ 3 \% .3 \% \mathrm{KNO}_{3}$ was prepared by dissolving $1.5 \mathrm{~g}$ of $\mathrm{KNO}_{3}$ in $500 \mathrm{~mL}$ of DDW. Seeds were soaked in the respective treatmental solutions for $24 \mathrm{~h}$ and re dried overnight (about $12 \mathrm{~h}$ ) and placed on petriplates. Seeds are moistened periodically with double distilled water.

Reducing sugars, Starch, alpha amylase were estimated at 48h (2 days), 96h (4 days) and $144 \mathrm{~h}$ (6 days) after soaking the seeds in different concentrations of $\mathrm{GA}_{3}$ and $\mathrm{KNO}_{3}$ treatments. By following standard protocols i.e; Nelson method for reducing sugars, Anthrone reagent method for estimation of starch and DNSA (Dinitro-salycilic acid) method for estimation of $\alpha$ - amylase content in rice seeds were recorded.

\section{Results and Discussion}

Reducing sugars ( $\left.\mathrm{mg} \mathrm{g}^{-1}\right)$

Maintenance of a higher amount of reducing sugar content is an important prerequisite for 
faster germination via enhanced metabolic activity of the embryo. Reducing sugar content was measured at 2, 4 and 6 DAT. A significant difference among varieties, treatments and their interaction was observed at all the stages and analyzed statistically and presented in table 1, 2 and 3 and Figure 1, 2 and 3. At 2 DAT it was observed that JGL 20171 (2.54) recorded significantly high reducing sugars followed by NLR 33671 (1.33). Among treatments $\mathrm{T}_{5}\left(\mathrm{GA}_{3} @ 200 \mathrm{ppm}\right.$ $+\mathrm{KNO}_{3} @ 3 \%$ ) (1.93) recorded significantly high reducing sugars followed by $\mathrm{T}_{6}\left(\mathrm{GA}_{3} @\right.$ $500 \mathrm{ppm}+\mathrm{KNO}_{3} @ 3 \%$ ) (1.79). $\mathrm{T}_{1}$ (control) (1.11) recorded lowest reducing sugars not only at this stage but also at 4 and 6 DAT (1.75 and 2.02).

At 4 and 6 DAT the genotypes MTU 1010 (2.86 and 3.28) and JGL 20171 (2.77 and 3.04) recorded significantly highest reducing sugars whereas the lowest value was recorded in MTU 1112 (1.9 and 2.14). Among treatments $\mathrm{T}_{5}\left(\mathrm{GA}_{3} @ 200 \mathrm{ppm}+\mathrm{KNO}_{3} @\right.$ 3\%) (3.07 and 3.19) and $\mathrm{T}_{6}\left(\mathrm{GA}_{3} @ 500\right.$ ppm $+\mathrm{KNO}_{3} @ 3 \%$ ) (2.96 and 3.35) showed better results. The interaction effect was found to be significant only at 2 DAT where in $\mathrm{V}_{3} \mathrm{~T}_{5}$ (3.20) recorded highest value.

A progressive increase in reducing sugars content was observed from 2 DAT to 6 DAT. MTU 1010, for example recorded the reducing sugar content of 1.30, 2.86 and 3.28 at 2,4 and 6 DAT respectively. The corresponding values for JGL 20171 were 2.54, 2.77 and 3.04 .

This increased availability of reducing sugars could be linked up with the activity of $\alpha$ amylase on breakdown of starch. Similar increase in reducing sugar content due to increased starch metabolism was also observed by Brain, 1959; Kuo and Yang, 1967; Basra et al., 2005 and Acharya et al., 2008.

\section{$\operatorname{Starch}\left(\mathrm{mg} \mathrm{g}^{-1}\right)$}

Starch represents the resource base of a seed. Higher starch content and its faster breakdown into sugars accelerate the metabolic processes of the embryo which leads to rapid cell division and cell expansion.

Data on influence of different seed priming treatments on starch content at 2, 4 and 6 DAT were presented in tables 4, 5 and 6 and Figure 4,5 and 6. From the data a gradual decrease in starch content was observed from 2 DAT to 6 DAT. Starch content in the genotype MTU 1010 for example at 2, 4 and 6 DAT was 41.2, 38.3, and 33.1 respectively. The corresponding values in MTU 1112 were 49.2, 46.2, and 42.1 respectively.

Irrespective of the genotype starch content decreased with time. Among the varieties MTU 1075 and MTU 1112 (50.1 and 49.2) recorded significantly higher and at par values of starch followed by MTU 7029 (43.5), MTU 1010 (41.2), NLR 3367 (41.0) and JGL 2017 (40.9) which were at par. MTU 1075 and MTU 1112 recorded highest starch content even at 4 and 6 DAT. This might be due to slow breakdown of starch in these genotypes.

Among treatments $\mathrm{T}_{8}\left(\mathrm{KNO}_{3} @ 3 \%\right)(50.1)$, $\mathrm{T}_{1}$ (Control) (49.8), $\mathrm{T}_{2}\left(\mathrm{GA}_{3} @ 200 \mathrm{ppm}\right)$ (48.3) and $\mathrm{T}_{3}\left(\mathrm{GA}_{3} @ 500\right.$ ppm) (46.9) recorded significantly higher and at par starch values where $\mathrm{T}_{5}\left(\mathrm{GA}_{3} @ 200 \mathrm{ppm}+\mathrm{KNO}_{3} @\right.$ $3 \%$ ) (38.8), $\mathrm{T}_{6}\left(\mathrm{GA}_{3} @ 500 \mathrm{ppm}+\mathrm{KNO}_{3} @ 3 \%\right)$ (39.4), $\mathrm{T}_{7}\left(\mathrm{GA}_{3} @ 1000 \mathrm{ppm}+\mathrm{KNO}_{3} @ 3 \%\right)$ (40) and $\mathrm{T}_{4}\left(\mathrm{GA}_{3} @ 1000 \mathrm{ppm}\right)$ (41.4) recorded significantly low and at par starch content at 2 DAT. This could be due to the influence of these treatments on faster breakdown of starch.

Almost a similar trend was observed at 4 and 6 DAT. The interaction effect was found to be non significant at all the stages. 
Table.1 Influence of different seed priming treatments and varieties on reducing sugars $\left(\mathrm{mg} \mathrm{g}^{-1}\right)$ at 2 days after treatment

\begin{tabular}{|l|c|c|c|c|c|c|c|c|c|}
\hline Variety & $\mathbf{T}_{\mathbf{1}}$ & $\mathbf{T}_{\mathbf{2}}$ & $\mathbf{T}_{\mathbf{3}}$ & $\mathbf{T}_{\mathbf{4}}$ & $\mathbf{T}_{\mathbf{5}}$ & $\mathbf{T}_{\mathbf{6}}$ & $\mathbf{T}_{\mathbf{7}}$ & $\mathbf{T}_{\mathbf{8}}$ & Mean \\
\hline MTU 1010 & 0.98 & 1.08 & 1.12 & 1.40 & 1.84 & 1.65 & 1.30 & 1.00 & $\mathbf{1 . 3 0}$ \\
\hline MTU 7029 & 1.00 & 1.10 & 1.20 & 1.34 & 1.83 & 1.56 & 1.48 & 1.10 & $\mathbf{1 . 3 3}$ \\
\hline JGL 20171 & 1.89 & 2.20 & 2.60 & 2.98 & 3.20 & 3.12 & 2.30 & 2.00 & $\mathbf{2 . 5 4}$ \\
\hline NLR 33671 & 0.89 & 1.10 & 1.30 & 1.40 & 1.67 & 1.60 & 1.50 & 1.20 & $\mathbf{1 . 3 3}$ \\
\hline MTU 1075 & 0.87 & 1.20 & 1.17 & 1.40 & 1.56 & 1.51 & 1.34 & 1.26 & $\mathbf{1 . 2 9}$ \\
\hline MTU 1112 & 1.00 & 1.20 & 1.20 & 1.23 & 1.45 & 1.30 & 1.32 & 1.30 & $\mathbf{1 . 2 5}$ \\
\hline Mean & $\mathbf{1 . 1 1}$ & $\mathbf{1 . 3 2}$ & $\mathbf{1 . 4 3}$ & $\mathbf{1 . 6 2}$ & $\mathbf{1 . 9 3}$ & $\mathbf{1 . 7 9}$ & $\mathbf{1 . 5 4}$ & $\mathbf{1 . 3 1}$ & \\
\hline & & & & & & & & \\
\hline & & & $\mathbf{V}$ & $\mathbf{T}$ & $\mathbf{V} \times \mathbf{T}$ & & & \\
\hline & & $\mathbf{C . D .}$ & $\mathbf{0 . 1 0}$ & $\mathbf{0 . 1 2}$ & $\mathbf{0 . 2 9}$ & & & \\
\hline & & $\mathbf{S E}(\mathbf{d})$ & 0.05 & 0.06 & 0.14 & & & \\
\hline & & $\mathbf{S E m} \pm$ & 0.04 & 0.04 & 0.10 & & & \\
\hline
\end{tabular}

\begin{tabular}{|l|l|}
\hline $\mathrm{T}_{1}:$ Control & $\mathrm{T}_{5}: \mathrm{GA}_{3} @ 200 \mathrm{ppm}+\mathrm{KNO}_{3} @ 3 \%$ \\
\hline $\mathrm{T}_{2}: \mathrm{GA}_{3} @ 200 \mathrm{ppm}$ & $\mathrm{T}_{6}: \mathrm{GA}_{3} @ 500 \mathrm{ppm}+\mathrm{KNO}_{3} @ 3 \%$ \\
\hline $\mathrm{T}_{3}: \mathrm{GA}_{3} @ 500 \mathrm{ppm}$ & $\mathrm{T}_{7}: \mathrm{GA}_{3} @ 1000 \mathrm{ppm}+\mathrm{KNO}_{3} @ 3 \%$ \\
\hline $\mathrm{T}_{4}: \mathrm{GA}_{3} @ 1000 \mathrm{ppm}$ & $\mathrm{T}_{8}: \mathrm{KNO}_{3} @ 3 \%$
\end{tabular}

Table.2 Influence of different seed priming treatments and varieties on reducing sugars $\left(\mathrm{mg} \mathrm{g}^{-1}\right)$ at 4 days after treatment

\begin{tabular}{|l|c|c|c|c|c|c|c|c|c|}
\hline Variety & $\mathbf{T}_{\mathbf{1}}$ & $\mathbf{T}_{\mathbf{2}}$ & $\mathbf{T}_{\mathbf{3}}$ & $\mathbf{T}_{\mathbf{4}}$ & $\mathbf{T}_{\mathbf{5}}$ & $\mathbf{T}_{\mathbf{6}}$ & $\mathbf{T}_{\mathbf{7}}$ & $\mathbf{T}_{\mathbf{8}}$ & Mean \\
\hline MTU 1010 & 2.34 & 2.56 & 2.60 & 3.15 & 3.40 & 3.23 & 3.10 & 2.50 & $\mathbf{2 . 8 6}$ \\
\hline MTU 7029 & 1.54 & 2.00 & 2.45 & 2.60 & 3.02 & 2.89 & 2.20 & 1.76 & $\mathbf{2 . 3 1}$ \\
\hline JGL 20171 & 2.19 & 2.42 & 2.59 & 3.13 & 3.26 & 3.18 & 3.04 & 2.35 & $\mathbf{2 . 7 7}$ \\
\hline NLR 33671 & 1.89 & 2.20 & 2.60 & 2.98 & 3.20 & 3.12 & 2.30 & 2.00 & $\mathbf{2 . 5 4}$ \\
\hline MTU 1075 & 1.34 & 1.80 & 2.30 & 2.54 & 3.00 & 2.80 & 2.10 & 1.70 & $\mathbf{2 . 2 0}$ \\
\hline MTU 1112 & 1.21 & 1.56 & 2.00 & 2.10 & 2.56 & 2.30 & 2.00 & 1.45 & $\mathbf{1 . 9 0}$ \\
\hline Mean & $\mathbf{1 . 7 5}$ & $\mathbf{2 . 0 9}$ & $\mathbf{2 . 4 2}$ & $\mathbf{2 . 7 5}$ & $\mathbf{3 . 0 7}$ & $\mathbf{2 . 9 2}$ & $\mathbf{2 . 4 6}$ & $\mathbf{1 . 9 6}$ & \\
\hline & & & & & & & & & \\
\hline & & & $\mathbf{V}$ & $\mathbf{T}$ & $\mathbf{V} \times \mathbf{T}$ & & & \\
\hline & & & C.D. & $\mathbf{0 . 1 6}$ & $\mathbf{0 . 1 9}$ & $\mathbf{N S}$ & & & \\
\hline & & SE(d) & 0.08 & 0.09 & 0.23 & & & \\
\hline & & SEm \pm & 0.06 & 0.07 & 0.16 & & & \\
\hline
\end{tabular}


Table.3 Influence of different seed priming treatments and varieties on reducing sugars $\left(\mathrm{mg} \mathrm{g}^{-1}\right)$ at 6 days after treatment

\begin{tabular}{|l|c|c|c|c|c|c|c|c|c|}
\hline Variety & $\mathbf{T}_{\mathbf{1}}$ & $\mathbf{T}_{\mathbf{2}}$ & $\mathbf{T}_{\mathbf{3}}$ & $\mathbf{T}_{\mathbf{4}}$ & $\mathbf{T}_{\mathbf{5}}$ & $\mathbf{T}_{\mathbf{6}}$ & $\mathbf{T}_{\mathbf{7}}$ & $\mathbf{T}_{\mathbf{8}}$ & Mean \\
\hline MTU 1010 & 2.67 & 3.00 & 3.30 & 3.56 & 3.56 & 3.78 & 3.40 & 2.98 & $\mathbf{3 . 2 8}$ \\
\hline MTU 7029 & 2.00 & 2.10 & 2.56 & 2.98 & 3.10 & 3.26 & 2.45 & 1.98 & $\mathbf{2 . 5 5}$ \\
\hline JGL 20171 & 2.40 & 2.64 & 2.98 & 3.30 & 3.40 & 3.54 & 3.25 & 2.80 & $\mathbf{3 . 0 4}$ \\
\hline NLR 33671 & 2.00 & 2.54 & 2.98 & 3.21 & 3.30 & 3.43 & 2.89 & 2.80 & $\mathbf{2 . 8 9}$ \\
\hline MTU 1075 & 1.56 & 1.90 & 2.40 & 2.78 & 3.00 & 3.10 & 2.45 & 1.90 & $\mathbf{2 . 3 9}$ \\
\hline MTU 1112 & 1.43 & 1.76 & 2.10 & 2.30 & 2.76 & 2.99 & 2.09 & 1.65 & $\mathbf{2 . 1 4}$ \\
\hline Mean & 2.01 & 2.32 & 2.72 & 3.02 & 3.19 & 3.35 & 2.76 & 2.35 & \\
\hline & & & & & & & & \\
\hline & & & $\mathbf{V}$ & $\mathbf{T}$ & $\mathbf{V} \times \mathbf{T}$ & & & \\
\hline & & $\mathbf{C . D .}$ & $\mathbf{0 . 1 8}$ & $\mathbf{0 . 2 1}$ & $\mathbf{N S}$ & & & \\
\hline & & $\mathbf{S E}(\mathbf{d})$ & 0.09 & 0.10 & 0.25 & & & \\
\hline & & $\mathbf{S E m} \pm$ & 0.06 & 0.07 & 0.18 & & &
\end{tabular}

\begin{tabular}{|l|l|}
\hline $\mathrm{T}_{1}:$ Control \\
\hline $\mathrm{T}_{2}: \mathrm{GA}_{3} @ 200 \mathrm{ppm}$ \\
\hline $\mathrm{T}_{3}: \mathrm{GA}_{3} @ 500 \mathrm{ppm}$ \\
\hline $\mathrm{T}_{4}: \mathrm{GA}_{3} @ 1000 \mathrm{ppm}$ \\
\hline
\end{tabular}

$\mathrm{T}_{5}: \mathrm{GA}_{3} @ 200 \mathrm{ppm}+\mathrm{KNO}_{3} @ 3 \%$

$\mathrm{T}_{6}: \mathrm{GA}_{3} @ 500 \mathrm{ppm}+\mathrm{KNO}_{3} @ 3 \%$

$\mathrm{T}_{7}: \mathrm{GA}_{3} @ 1000 \mathrm{ppm}+\mathrm{KNO}_{3} @ 3 \%$

$\mathrm{T}_{8}: \mathrm{KNO}_{3} @ 3 \%$

Table.4 Influence of different seed priming treatments and varieties on starch (mg g-1) at 2 days after treatment

\begin{tabular}{|l|c|c|c|c|c|c|c|c|c|}
\hline Variety & $\mathbf{T}_{\mathbf{1}}$ & $\mathbf{T}_{\mathbf{2}}$ & $\mathbf{T}_{\mathbf{3}}$ & $\mathbf{T}_{\mathbf{4}}$ & $\mathbf{T}_{\mathbf{5}}$ & $\mathbf{T}_{\mathbf{6}}$ & $\mathbf{T}_{\mathbf{7}}$ & $\mathbf{T}_{\mathbf{8}}$ & $\mathbf{M e a n}$ \\
\hline MTU 1010 & 46.2 & 43.1 & 41.2 & 40.8 & 33.6 & 38.6 & 37.5 & 48.5 & $\mathbf{4 1 . 2}$ \\
\hline MTU 7029 & 51.0 & 53.0 & 46.0 & 39.0 & 38.5 & 36.0 & 33.5 & 51.0 & $\mathbf{4 3 . 5}$ \\
\hline JGL 20171 & 43.9 & 43.8 & 42.5 & 37.5 & 38.5 & 39.7 & 36.5 & 44.8 & $\mathbf{4 0 . 9}$ \\
\hline NLR 33671 & 47.1 & 45.2 & 42.5 & 39.8 & 33.6 & 34.5 & 39.4 & 46.2 & $\mathbf{4 1 . 0}$ \\
\hline MTU 1075 & 56.5 & 54.8 & 56.1 & 44.5 & 43.9 & 44.1 & 45.3 & 55.5 & $\mathbf{5 0 . 1}$ \\
\hline MTU 1112 & 53.8 & 49.8 & 52.9 & 46.9 & 44.5 & 43.5 & 47.8 & 54.6 & $\mathbf{4 9 . 2}$ \\
\hline Mean & 49.8 & 48.3 & 46.9 & 41.4 & 38.8 & 39.4 & 40.0 & 50.1 & \\
\hline & & & & & & & & \\
\hline & & & $\mathbf{V}$ & $\mathbf{T}$ & $\mathbf{V} \times \mathbf{T}$ & & & \\
\hline & & $\mathbf{C . D .}$ & $\mathbf{2 . 9 0}$ & $\mathbf{3 . 3 4}$ & $\mathbf{N S}$ & & & \\
\hline & & $\mathbf{S E}(\mathbf{d})$ & 1.46 & 1.68 & 4.12 & & & \\
\hline & & $\mathbf{S E m} \pm$ & 1.03 & 1.19 & 2.91 & & & \\
\hline
\end{tabular}


Table.5 Influence of different seed priming treatments and varieties on starch $\left(\mathrm{mg} \mathrm{g}^{-1}\right)$ at 4 days after treatment

\begin{tabular}{|l|c|c|c|c|c|c|c|c|c|}
\hline Variety & $\mathbf{T}_{\mathbf{1}}$ & $\mathbf{T}_{\mathbf{2}}$ & $\mathbf{T}_{\mathbf{3}}$ & $\mathbf{T}_{\mathbf{4}}$ & $\mathbf{T}_{\mathbf{5}}$ & $\mathbf{T}_{\mathbf{6}}$ & $\mathbf{T}_{\mathbf{7}}$ & $\mathbf{T}_{\mathbf{8}}$ & $\mathbf{M e a n}$ \\
\hline MTU 1010 & 44.6 & 45.7 & 42.5 & 31.8 & 31.6 & 34.5 & 32.5 & 43.5 & $\mathbf{3 8 . 3}$ \\
\hline MTU 7029 & 51.2 & 42.5 & 45.5 & 33.8 & 35.6 & 36.8 & 40.1 & 45.5 & $\mathbf{4 1 . 4}$ \\
\hline JGL 20171 & 43.5 & 41.7 & 42.8 & 30.6 & 34.5 & 36.2 & 33.5 & 48.2 & $\mathbf{3 8 . 9}$ \\
\hline NLR 33671 & 45.6 & 45.8 & 43.5 & 34.8 & 36.9 & 32.8 & 37.4 & 44.8 & $\mathbf{4 0 . 2}$ \\
\hline MTU 1075 & 44.6 & 48.5 & 43.2 & 36.5 & 37.8 & 36.5 & 38.6 & 47.2 & $\mathbf{4 1 . 6}$ \\
\hline MTU 1112 & 56.8 & 55.6 & 53.2 & 38.2 & 36.3 & 37.1 & 36.5 & 55.7 & $\mathbf{4 6 . 2}$ \\
\hline Mean & $\mathbf{4 7 . 7}$ & $\mathbf{4 6 . 6}$ & $\mathbf{4 5 . 1}$ & $\mathbf{3 4 . 3}$ & $\mathbf{3 5 . 5}$ & $\mathbf{3 5 . 7}$ & $\mathbf{3 6 . 4}$ & $\mathbf{4 7 . 5}$ & \\
\hline & & & & & & & & & \\
\hline & & & $\mathbf{V}$ & $\mathbf{T}$ & $\mathbf{V} \times \mathbf{T}$ & & & \\
\hline & & $\mathbf{C . D .}$ & $\mathbf{2 . 7 0}$ & $\mathbf{3 . 1 2}$ & $\mathbf{N S}$ & & & \\
\hline & & $\mathbf{S E}(\mathbf{d})$ & 1.36 & 1.57 & 3.84 & & & \\
\hline & & $\mathbf{S E m} \pm$ & 0.96 & 1.11 & 2.72 & & &
\end{tabular}

\begin{tabular}{|l|l|}
\hline $\mathrm{T}_{1}:$ Control & $\mathrm{T}_{5}: \mathrm{GA}_{3} @ 200 \mathrm{ppm}+\mathrm{KNO}_{3} @ 3 \%$ \\
\hline $\mathrm{T}_{2}: \mathrm{GA}_{3} @ 200 \mathrm{ppm}$ & $\mathrm{T}_{6}: \mathrm{GA}_{3} @ 500 \mathrm{ppm}+\mathrm{KNO}_{3} @ 3 \%$ \\
\hline $\mathrm{T}_{3}: \mathrm{GA}_{3} @ 500 \mathrm{ppm}$ & $\mathrm{T}_{7}: \mathrm{GA}_{3} @ 1000 \mathrm{ppm}+\mathrm{KNO}_{3} @ 3 \%$ \\
\hline $\mathrm{T}_{4}: \mathrm{GA}_{3} @ 1000 \mathrm{ppm}$ & $\mathrm{T}_{8}: \mathrm{KNO}_{3} @ 3 \%$
\end{tabular}

Table.6 Influence of different seed priming treatments and varieties on Starch $\left(\mathrm{mg} \mathrm{g}^{-1}\right)$ at 6 days after treatment

\begin{tabular}{|l|c|c|c|c|c|c|c|c|c|}
\hline Variety & $\mathbf{T}_{\mathbf{1}}$ & $\mathbf{T}_{\mathbf{2}}$ & $\mathbf{T}_{\mathbf{3}}$ & $\mathbf{T}_{\mathbf{4}}$ & $\mathbf{T}_{\mathbf{5}}$ & $\mathbf{T}_{\mathbf{6}}$ & $\mathbf{T}_{\mathbf{7}}$ & $\mathbf{T}_{\mathbf{8}}$ & Mean \\
\hline MTU 1010 & 38.3 & 37.4 & 35.5 & 31.6 & 29.4 & 28.6 & 27.8 & 36.5 & $\mathbf{3 3 . 1}$ \\
\hline MTU 7029 & 40.5 & 41.2 & 38.9 & 29.8 & 30.5 & 27.8 & 31.5 & 35.7 & $\mathbf{3 4 . 5}$ \\
\hline JGL 20171 & 38.7 & 35.8 & 36.3 & 26.2 & 24.8 & 24.5 & 25.8 & 39.5 & $\mathbf{3 1 . 5}$ \\
\hline NLR 33671 & 44.6 & 40.8 & 43.9 & 26.4 & 27.3 & 29.8 & 28.7 & 42.7 & $\mathbf{3 5 . 5}$ \\
\hline MTU 1075 & 51.3 & 45.9 & 47.8 & 34.2 & 30.5 & 35.2 & 33.8 & 46.7 & $\mathbf{4 0 . 7}$ \\
\hline MTU 1112 & 52.4 & 50.6 & 42.3 & 36.3 & 38.4 & 34.8 & 36.8 & 45.1 & $\mathbf{4 2 . 1}$ \\
\hline Mean & $\mathbf{4 4 . 3}$ & $\mathbf{4 2 . 0}$ & $\mathbf{4 0 . 8}$ & $\mathbf{3 0 . 8}$ & $\mathbf{3 0 . 2}$ & $\mathbf{3 0 . 1}$ & $\mathbf{3 0 . 7}$ & $\mathbf{4 1 . 0}$ & \\
\hline & & & & & & & & \\
\hline & & & $\mathbf{V}$ & $\mathbf{T}$ & $\mathbf{V} \times \mathbf{T}$ & & & \\
\hline & & $\mathbf{C . D .}$ & $\mathbf{2 . 4 2}$ & $\mathbf{2 . 7 9}$ & $\mathbf{N S}$ & & & \\
\hline & & $\mathbf{S E}(\mathbf{d})$ & 1.22 & 1.41 & 3.44 & & & \\
\hline & & $\mathbf{S E m} \pm$ & 0.86 & 0.99 & 2.43 & & & \\
\hline & & & & & & &
\end{tabular}


Table.7 Influence of different seed priming treatments and varieties on alpha amylase $\left(\mathrm{mg} \mathrm{g}^{-1}\right)$ at 2 days after treatment

\begin{tabular}{|l|c|c|c|c|c|c|c|c|c|}
\hline Variety & $\mathbf{T}_{\mathbf{1}}$ & $\mathbf{T}_{\mathbf{2}}$ & $\mathbf{T}_{\mathbf{3}}$ & $\mathbf{T}_{\mathbf{4}}$ & $\mathbf{T}_{\mathbf{5}}$ & $\mathbf{T}_{\mathbf{6}}$ & $\mathbf{T}_{\mathbf{7}}$ & $\mathbf{T}_{\mathbf{8}}$ & Mean \\
\hline MTU 1010 & 1.9 & 1.9 & 1.8 & 2.4 & 2.2 & 2.3 & 2.3 & 1.9 & $\mathbf{2 . 1}$ \\
\hline MTU 7029 & 1.7 & 2.0 & 1.7 & 2.2 & 2.1 & 2.2 & 2.2 & 1.9 & $\mathbf{2 . 0}$ \\
\hline JGL 20171 & 2.0 & 2.1 & 2.0 & 2.4 & 2.3 & 2.4 & 2.4 & 2.0 & $\mathbf{2 . 2}$ \\
\hline NLR 33671 & 1.7 & 1.5 & 1.5 & 2.2 & 2.3 & 2.3 & 2.1 & 1.7 & $\mathbf{1 . 9}$ \\
\hline MTU 1075 & 0.9 & 0.9 & 0.8 & 1.3 & 1.4 & 1.3 & 1.3 & 0.9 & $\mathbf{1 . 1}$ \\
\hline MTU 1112 & 0.7 & 0.8 & 0.7 & 1.3 & 1.3 & 1.2 & 1.1 & 0.8 & $\mathbf{1 . 0}$ \\
\hline Mean & $\mathbf{1 . 5}$ & $\mathbf{1 . 5}$ & $\mathbf{1 . 4}$ & $\mathbf{1 . 9}$ & $\mathbf{1 . 9}$ & $\mathbf{2 . 0}$ & $\mathbf{1 . 9}$ & $\mathbf{1 . 5}$ & \\
\hline & & & & & & & & & \\
\hline & & & $\mathbf{V}$ & $\mathbf{T}$ & $\mathbf{V} \times \mathbf{T}$ & & & \\
\hline & & $\mathbf{C . D .}$ & $\mathbf{0 . 1 1}$ & $\mathbf{0 . 1 3}$ & $\mathbf{N S}$ & & & \\
\hline & & $\mathbf{S E}(\mathbf{d})$ & 0.06 & 0.07 & 0.16 & & & \\
\hline & & $\mathbf{S E m} \pm$ & 0.04 & 0.05 & 0.11 & & & \\
\hline
\end{tabular}

\begin{tabular}{|l|l|}
\hline $\mathrm{T}_{1}:$ Control & $\mathrm{T}_{5}: \mathrm{GA}_{3} @ 200 \mathrm{ppm}+\mathrm{KNO}_{3} @ 3 \%$ \\
\hline $\mathrm{T}_{2}: \mathrm{GA}_{3} @ 200 \mathrm{ppm}$ & $\mathrm{T}_{6}: \mathrm{GA}_{3} @ 500 \mathrm{ppm}+\mathrm{KNO}_{3} @ 3 \%$ \\
\hline $\mathrm{T}_{3}: \mathrm{GA}_{3} @ 500 \mathrm{ppm}$ & $\mathrm{T}_{7}: \mathrm{GA}_{3} @ 1000 \mathrm{ppm}+\mathrm{KNO}_{3} @ 3 \%$ \\
\hline $\mathrm{T}_{4}: \mathrm{GA}_{3} @ 1000 \mathrm{ppm}$ & $\mathrm{T}_{8}: \mathrm{KNO}_{3} @ 3 \%$
\end{tabular}

Table.8 Influence of different seed priming treatments and varieties on alpha amylase $\left(\mathrm{mg} \mathrm{g}^{-1}\right)$ at 4 days after treatment

\begin{tabular}{|l|c|c|c|c|c|c|c|c|c|}
\hline Variety & $\mathbf{T}_{\mathbf{1}}$ & $\mathbf{T}_{\mathbf{2}}$ & $\mathbf{T}_{\mathbf{3}}$ & $\mathbf{T}_{\mathbf{4}}$ & $\mathbf{T}_{\mathbf{5}}$ & $\mathbf{T}_{\mathbf{6}}$ & $\mathbf{T}_{\mathbf{7}}$ & $\mathbf{T}_{\mathbf{8}}$ & Mean \\
\hline MTU 1010 & 2.1 & 2.2 & 2.4 & 2.7 & 2.7 & 2.8 & 2.8 & 2.2 & $\mathbf{2 . 5}$ \\
\hline MTU 7029 & 2.0 & 2.0 & 2.5 & 2.3 & 2.5 & 2.6 & 2.6 & 2.1 & $\mathbf{2 . 3}$ \\
\hline JGL 20171 & 2.5 & 2.4 & 2.4 & 2.6 & 2.8 & 2.8 & 2.9 & 2.3 & $\mathbf{2 . 6}$ \\
\hline NLR 33671 & 1.8 & 1.6 & 2.0 & 2.8 & 2.6 & 2.3 & 2.5 & 1.9 & $\mathbf{2 . 2}$ \\
\hline MTU 1075 & 1.0 & 0.9 & 1.0 & 1.4 & 1.4 & 1.9 & 1.8 & 1.2 & $\mathbf{1 . 3}$ \\
\hline MTU 1112 & 0.8 & 0.8 & 1.0 & 1.4 & 1.5 & 1.6 & 1.3 & 0.8 & $\mathbf{1 . 1}$ \\
\hline Mean & $\mathbf{1 . 7}$ & $\mathbf{1 . 6}$ & $\mathbf{1 . 9}$ & $\mathbf{2 . 2}$ & $\mathbf{2 . 2}$ & $\mathbf{2 . 3}$ & $\mathbf{2 . 3}$ & $\mathbf{1 . 7}$ & \\
\hline & & & & & & & & & \\
\hline & & & $\mathbf{V}$ & $\mathbf{T}$ & $\mathbf{V} \times \mathbf{T}$ & & & \\
\hline & & $\mathbf{C . D .}$ & $\mathbf{0 . 1 3}$ & $\mathbf{0 . 1 6}$ & $\mathbf{N S}$ & & & \\
\hline & & $\mathbf{S E}(\mathbf{d})$ & 0.07 & 0.08 & 0.19 & & & \\
\hline & & $\mathbf{S E m} \pm$ & 0.05 & 0.06 & 0.14 & & &
\end{tabular}


Table.9 Influence of different seed priming treatments and varieties on Alpha amylase $\left(\mathrm{mg} \mathrm{g}^{-1}\right)$ at 6 days after treatment

\begin{tabular}{|l|c|c|c|c|c|c|c|c|c|}
\hline Variety & $\mathbf{T}_{\mathbf{1}}$ & $\mathbf{T}_{\mathbf{2}}$ & $\mathbf{T}_{\mathbf{3}}$ & $\mathbf{T}_{\mathbf{4}}$ & $\mathbf{T}_{\mathbf{5}}$ & $\mathbf{T}_{\mathbf{6}}$ & $\mathbf{T}_{\mathbf{7}}$ & $\mathbf{T}_{\mathbf{8}}$ & Mean \\
\hline MTU 1010 & 1.6 & 1.5 & 1.6 & 2.1 & 2.2 & 2.5 & 2.7 & 1.2 & $\mathbf{1 . 9}$ \\
\hline MTU 7029 & 1.4 & 1.7 & 1.4 & 2.0 & 1.8 & 2.0 & 2.1 & 1.3 & $\mathbf{1 . 7}$ \\
\hline JGL 20171 & 1.6 & 1.8 & 1.9 & 2.0 & 2.0 & 2.1 & 1.9 & 1.2 & $\mathbf{1 . 8}$ \\
\hline NLR 33671 & 1.2 & 1.6 & 0.9 & 1.8 & 2.1 & 2.3 & 1.8 & 0.8 & $\mathbf{1 . 6}$ \\
\hline MTU 1075 & 0.9 & 0.8 & 0.7 & 1.2 & 1.6 & 1.6 & 1.7 & 1.0 & $\mathbf{1 . 2}$ \\
\hline MTU 1112 & 0.7 & 0.9 & 1.2 & 1.2 & 1.4 & 1.3 & 1.4 & 0.7 & $\mathbf{1 . 1}$ \\
\hline Mean & $\mathbf{1 . 2}$ & $\mathbf{1 . 3}$ & $\mathbf{1 . 3}$ & $\mathbf{1 . 7}$ & $\mathbf{1 . 9}$ & $\mathbf{2 . 0}$ & $\mathbf{1 . 9}$ & $\mathbf{1 . 0}$ & \\
\hline & & & & & & & & & \\
\hline & & & $\mathbf{V}$ & $\mathbf{T}$ & $\mathbf{V} \times \mathbf{T}$ & & & \\
\hline & & C.D. & $\mathbf{0 . 1 0}$ & $\mathbf{0 . 1 2}$ & $\mathbf{0 . 2 9}$ & & & \\
\hline & & SE(d) & 0.05 & 0.06 & 0.15 & & & \\
\hline & & SEm \pm & 0.04 & 0.04 & 0.10 & & &
\end{tabular}

\begin{tabular}{|l|l|}
\hline $\mathrm{T}_{1}:$ Control & $\mathrm{T}_{5}: \mathrm{GA}_{3} @ 200 \mathrm{ppm}+\mathrm{KNO}_{3} @ 3 \%$ \\
\hline $\mathrm{T}_{2}: \mathrm{GA}_{3} @ 200 \mathrm{ppm}$ & $\mathrm{T}_{6}: \mathrm{GA}_{3} @ 500 \mathrm{ppm}+\mathrm{KNO}_{3} @ 3 \%$ \\
\hline $\mathrm{T}_{3}: \mathrm{GA}_{3} @ 500 \mathrm{ppm}$ & $\mathrm{T}_{7}: \mathrm{GA}_{3} @ 1000 \mathrm{ppm}+\mathrm{KNO}_{3} @ 3 \%$ \\
\hline $\mathrm{T}_{4}: \mathrm{GA}_{3} @ 1000 \mathrm{ppm}$ & $\mathrm{T}_{8}: \mathrm{KNO}_{3} @ 3 \%$ \\
\hline
\end{tabular}

Fig.1 Influence of different seed priming treatments and varieties on reducing sugars $\left(\mathrm{mg} \mathrm{g}^{-1}\right)$ at 2 days after treatment

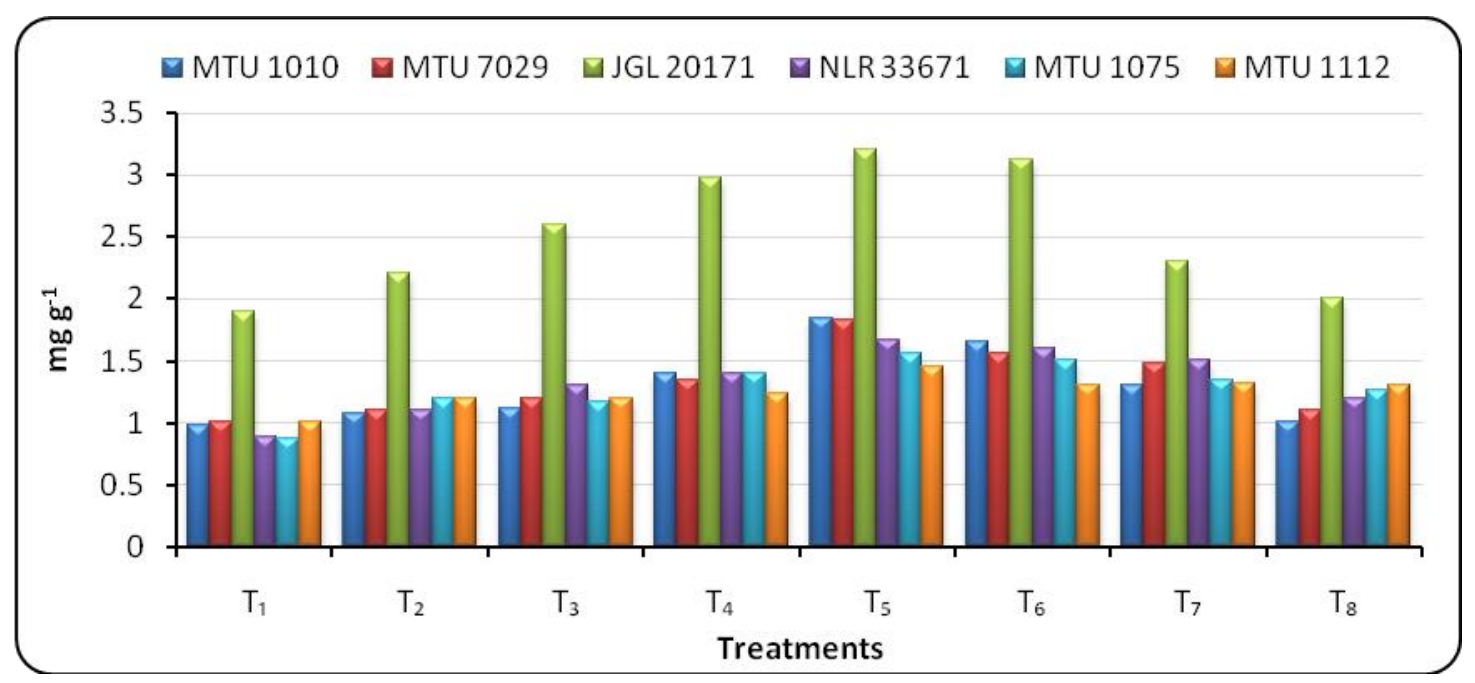


Fig.2 Influence of different seed priming treatments and varieties on reducing sugars $\left(\mathrm{mg} \mathrm{g}^{-1}\right)$ at 4 days after treatment

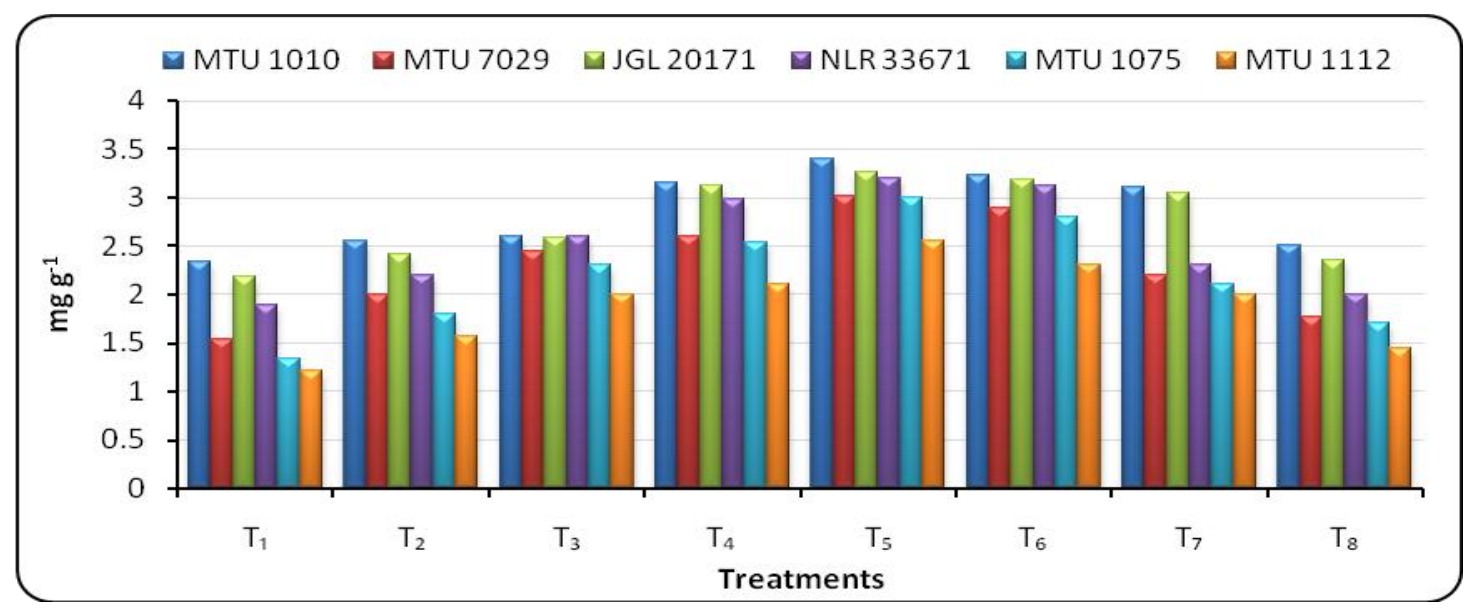

Fig.3 Influence of different seed priming treatments and varieties on reducing sugars (mg g-1) at 6 days after treatment

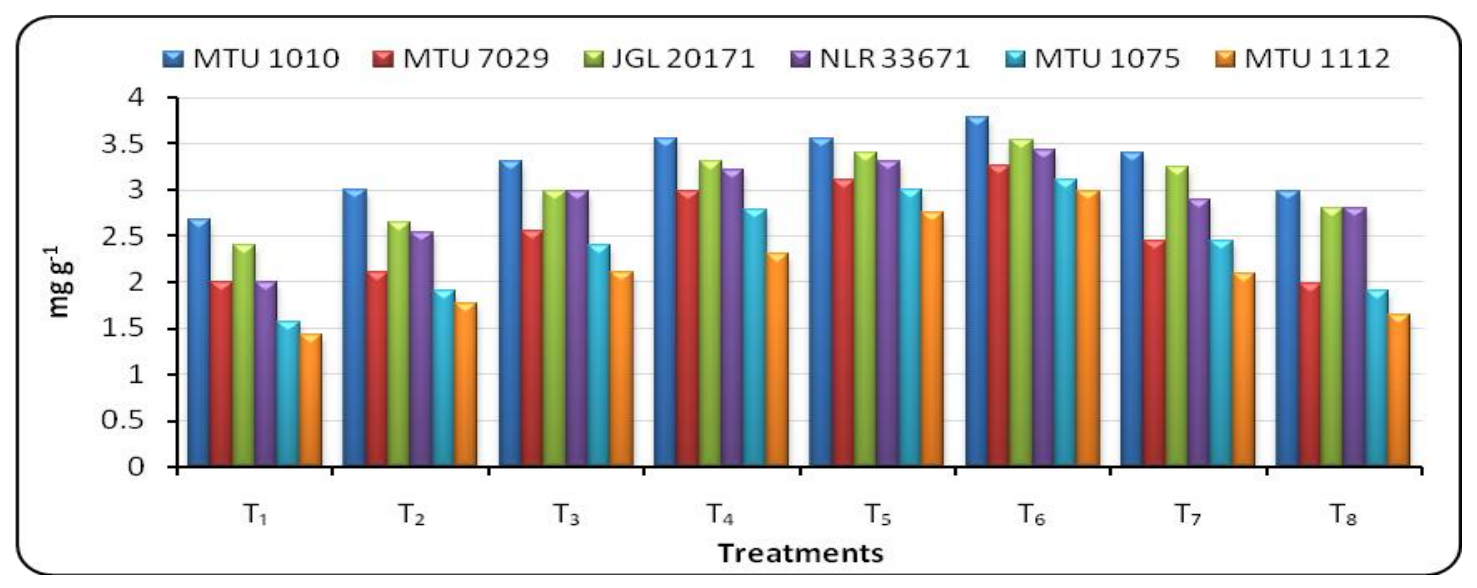

Fig.4 Influence of different seed priming treatments and varieties on starch $\left(\mathrm{mg} \mathrm{g}^{-1}\right)$ index at 2 days after treatment

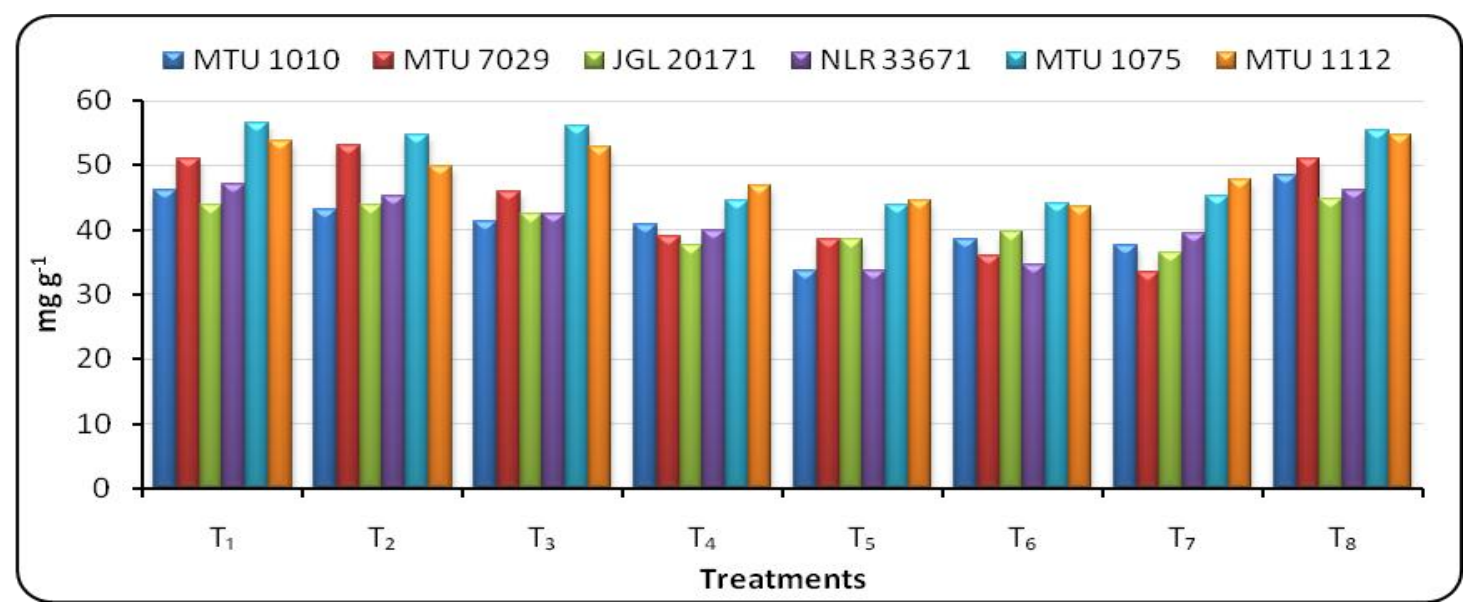


Fig.5 Influence of different seed priming treatments and varieties on starch $\left(\mathrm{mg} \mathrm{g}^{-1}\right)$ at 4 days after treatment

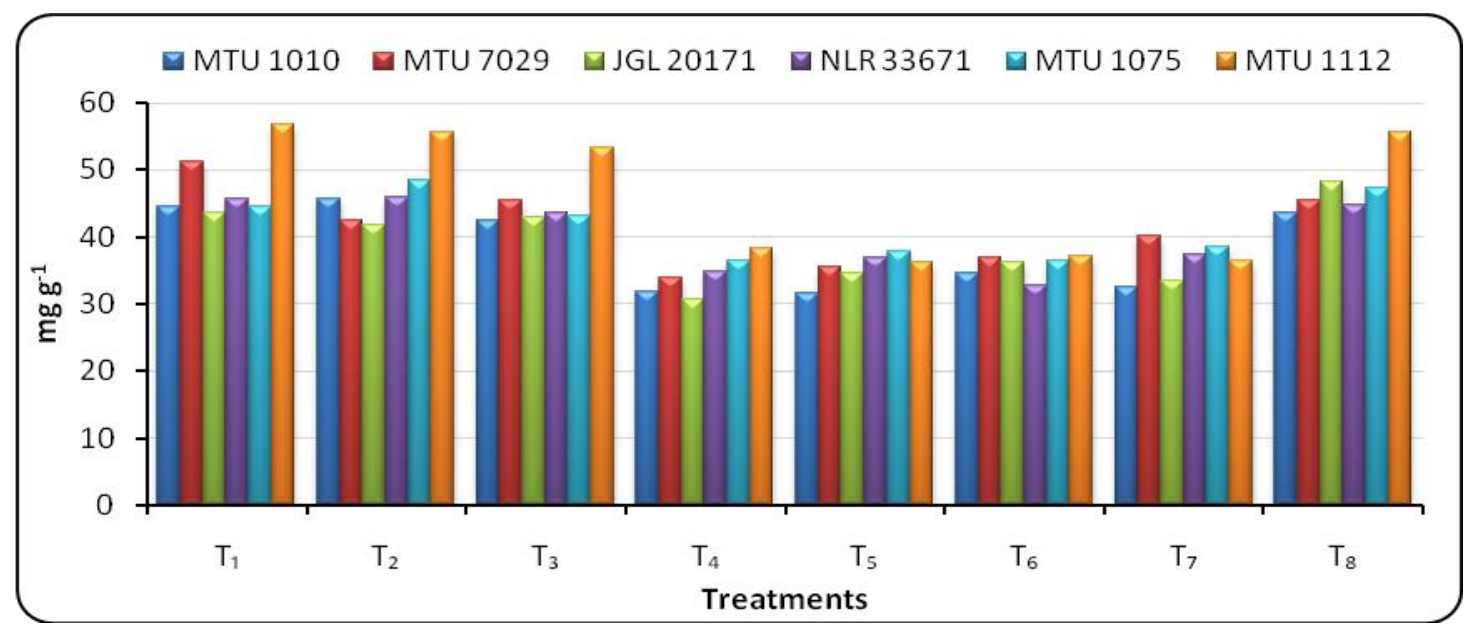

Fig.6 Influence of different seed priming treatments and varieties on starch (mg g-1) at 6 days after treatment

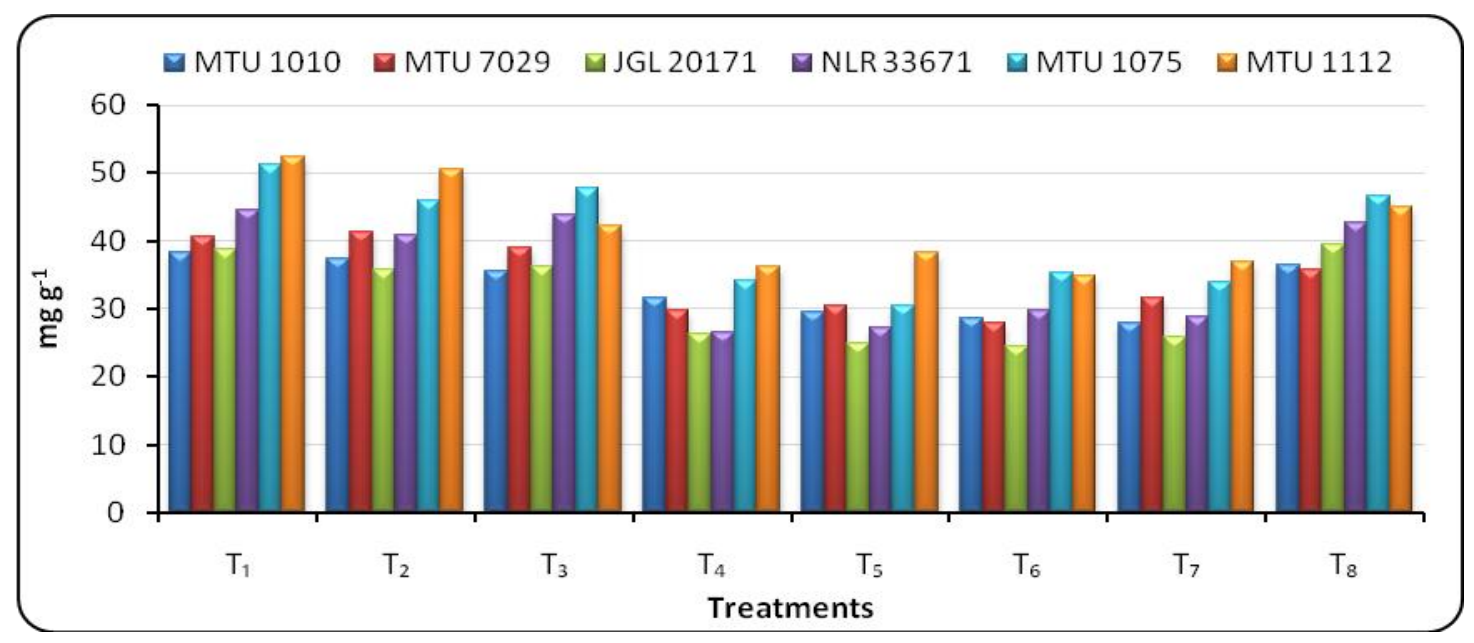

Fig.7 Influence of different seed priming treatments and varieties on alpha amylase $\left(\mathrm{mg} \mathrm{g}^{-1}\right)$ at 2 days after treatment

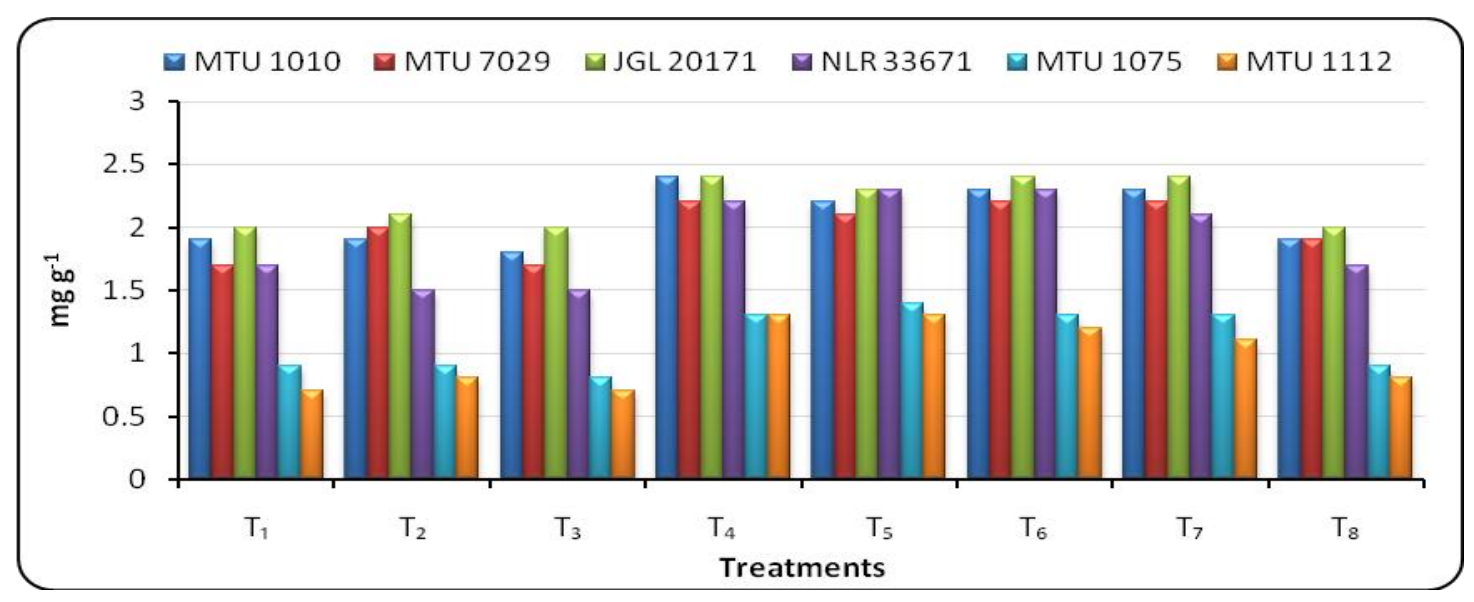


Fig.8 Influence of different seed priming treatments and varieties on alpha amylase $\left(\mathrm{mg} \mathrm{g}^{-1}\right)$ at 4 days after treatment

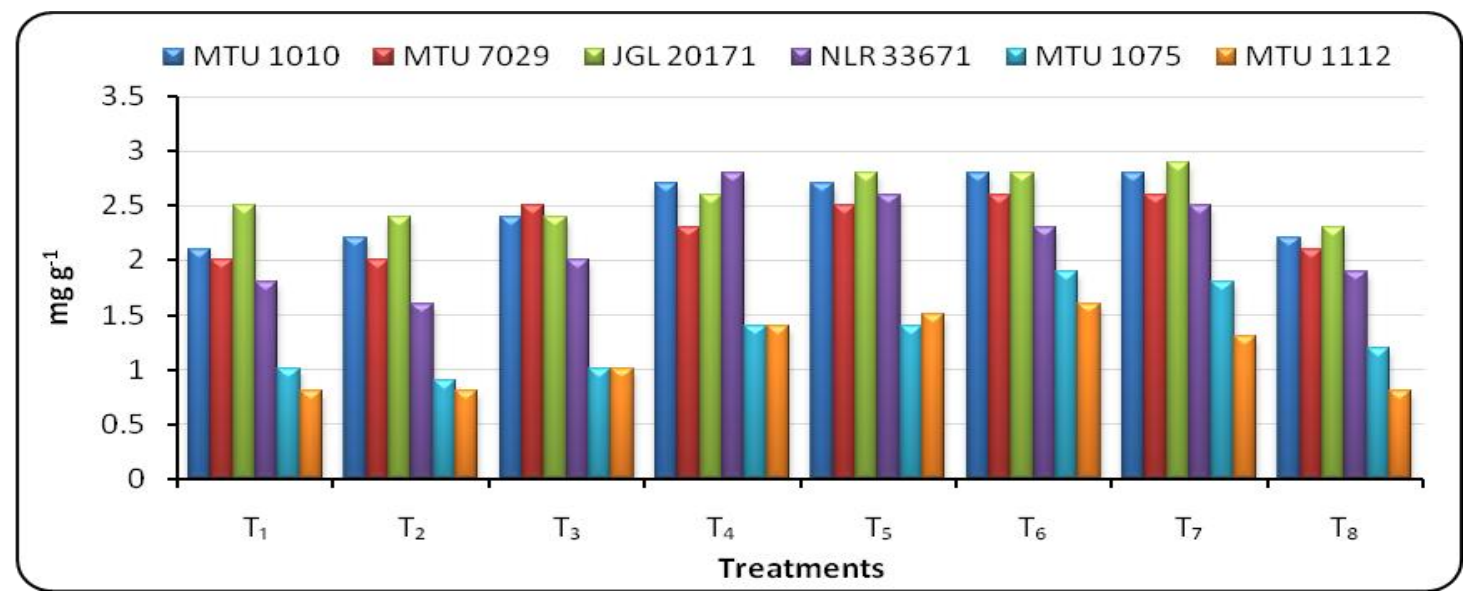

Fig.9 Influence of different seed priming treatments and varieties on alpha amylase $\left(\mathrm{mg} \mathrm{g}^{-1}\right)$ at 6 days after treatment

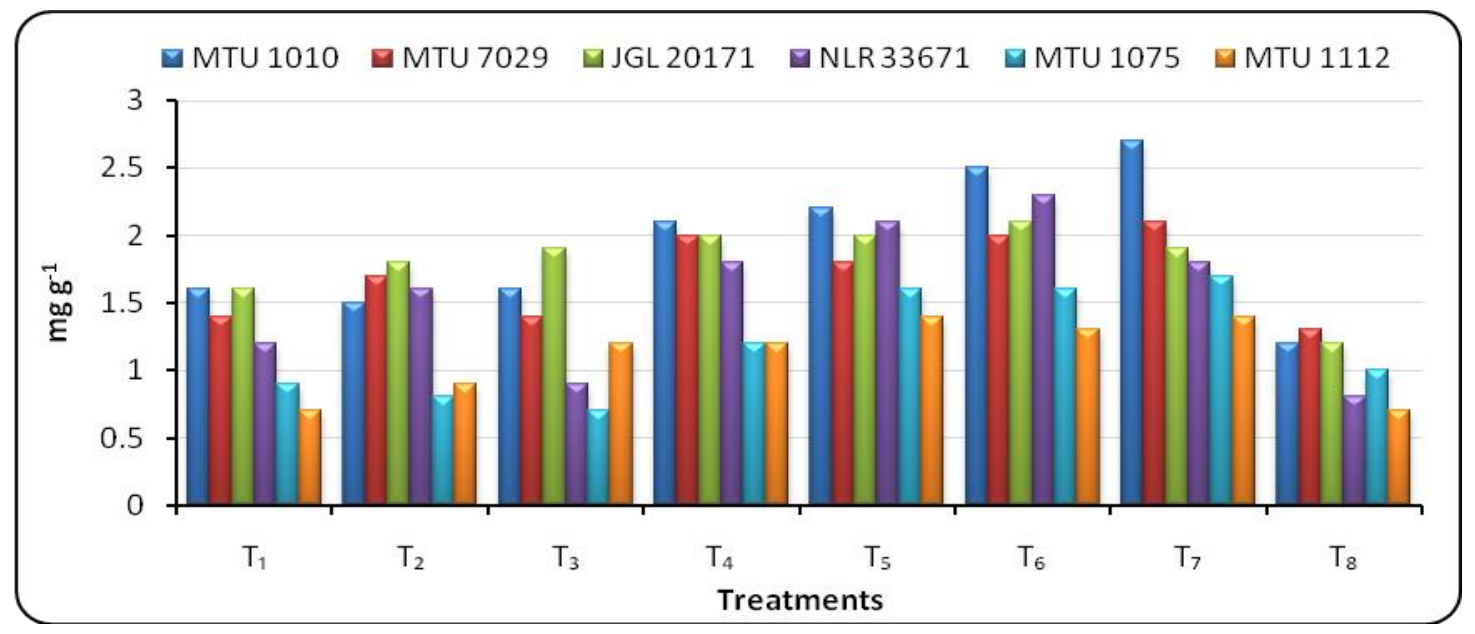

Robolledo et al., (2012) also reported that stored starch is an important factor for growth and maintenance to balance vigour and moisture stress tolerance in aerobic condition of rice. Further, Luquet et al., (2012) suggested that besides morphological traits, non structural carbohydrates particularly starch could be used as relevant marker to screen genotypes for early seedling vigour in aerobic rice.

\section{Alpha - amylase ( $\left.\mathrm{mg} \mathrm{g}^{-1}\right)$}

De-nova synthesis of $\alpha$-amylase in aleurone layer is believed to be essential for seed germination in cereal grains, which is tightly regulated by $\mathrm{GA}_{3}$ synthesized in the embryonic layer.

Data on influence of different seed priming treatments on different aerobic rice genotypes with respect to $\alpha$ - amylase content at 2, 4 and 6 DAT was presented in table 7, 8 and 9 and Figure 7, 8 and 9 respectively.

The results revealed a gradual increase in $\alpha$ amylase content from 2 DAT to 4 DAT. Later it was decreased at 6 DAT. Among varieties JGL 20171 (2.2, 2.6 and 1.8) and MTU 1010 (2.1,2.5 and 1.9) were found to consist 
significantly highest and at par $\alpha$-amylase content whereas MTU 1112 (1.0) and MTU 1075 (1.1) recorded significantly lowest and at par values at 2, 4 and 6 DAT. It indicates the genotypic difference in $\alpha$-amylase content.

This higher $\alpha$-amylase content might be the reason for higher seedling vigour index in these genotypes. This genotypic character was seem to be enhanced further with different treatments.

Among treatments $\mathrm{T}_{6}\left(\mathrm{GA}_{3} @ 500\right.$ ppm + $\mathrm{KNO}_{3} @ 3 \%$ ) (2.0) recorded significantly highest $\alpha$-amylase content which was at par with $\mathrm{T}_{5}\left(\mathrm{GA}_{3} @ 200 \mathrm{ppm}+\mathrm{KNO}_{3} @ 3 \%\right)$ (1.9), $\mathrm{T}_{7}\left(\mathrm{GA}_{3} @ 1000 \mathrm{ppm}+\mathrm{KNO}_{3} @\right.$ $3 \%)(1.9)$ and $\mathrm{T}_{4}\left(\mathrm{GA}_{3} @ 1000\right.$ ppm) (1.9).

$\mathrm{T}_{5}\left(1.9,2.2\right.$ and 1.9) and $\mathrm{T}_{6}(2.0,2.3$ and 2.0) recorded highest $\alpha$-amylase content at all the stages whereas $\mathrm{T}_{8}$ (1.0) followed by $\mathrm{T}_{1}$ (1.2) recorded significantly lowest values at 6 DAT. This could explain the role of $\mathrm{GA}_{3}$ either alone or in combination in enhancing $\alpha$ amylase content.

The interaction effect was found to be non significant at 2 and 4 DAT. However, a significant interaction was observed at 6 DAT. Where in $\mathrm{V}_{1} \mathrm{~T}_{7}$ (2.7) recorded significantly highest $\alpha$-amylase content and the lowest was recorded in $\mathrm{V}_{6} \mathrm{~T}_{1}(0.7)$.

\section{References}

Acharya, U.T., Prakash, L and Prathapasenan, G. 2008. Effect of gibberellic acid on seedling growth and carbohydrate metabolism during germination of rice (Oryza sativa L. var. GR-3) under saline condition. Journal of Agronomy and Crop Science. 165(1): 6-13 70.

Basra, S.M.A., Farooq, M., Tabassam, R and Ahmad, N. 2005. Physiological and biochemical aspects of pre-sowing seed treatments in fine rice (Oryza sativa L.). Seed Science and Technology. 33: 623628.

Brain, P. W. 1959.Effects of gibberellins on growth and development. Biological Review. 34: 37-84.

Foolad, M., Subbiah, P., Zhang, L. 2007. Common QTL affect the rate of tomato seed germination under different stress and non stress conditions. International Journal of Plant Genomics.1-10.

IRRI, 2017. http://ricestat.irri.org

Jayadeva, H.M., Bhairappanavar, S.T., Hugar, A.Y., Rangaswamy, B.R., Mallikarjun, G.B., Malleshappa, C and Naik, D.C. 2011. Integrated weed management in aerobic rice (Oryza sativa L.). Agriculture Science Digest. 31(1): 5861.

Kuo, T.T and Yang, S.E. 1967. Physiology of Bakane disease. 1. Effect of $\mathrm{GA}_{3}$ on the metabolic changes in germinating rice seeds. Botanical Bulletin of Academic Science. 8:199-207.

Luquet, D. Soulie, J. Rebolledo, M. Rouan, L. Clement-Vidal, A and Dingkuhn, $\mathrm{M}$. 2012. Developmental dynamics and early growthvigour in rice 2 . Modelling genetic diversity using Ecomeristem.Journal of Agronomy and Crop Science. 198:385-398.

Nelson, N. 1944.A photometric adaptation of the Somogy's method for determination of glucose. Journal of biochemistry.153: 375-380.

Nie, L., Peng, S., Chen, M., Shah, F., Huang, J., Cui, K and Xiang, J. 2012. Aerobic rice for water-saving agriculture. $A$ review of Agronomy and Sustainable Development. 32:411-418.

Qin, Y., Liu, S., Guo, Y., Liu, Q and Zou, J. 2010.Methane and nitrous oxide emissions from organic and conventional rice cropping systems in Southeast China. Biology and Fertility of Soils. 46: 825-834. 
Rebolledo, M.C., Dingkhun, A.C.V., Vidal, A.C., Rouan, L and Luquet, D. 2012. Phenomics of rice early vigour and drought response: Are sugar related and morphogenetic traits relevant? The Rice Journal. 5:22.

Sadasivam, S and Manickam, A.1996. Biochemical methods for agricultural sciences. Wiley Eastern Ltd. New Delhi.

Singh, A.K., Pal, S., Datta, S.P., and Rattan, R.K. 2008. Diagnosis and amelioration of iron deficiency under aerobic rice. Journal of Plant Nutrition. 31(5):919940.

Sunil, C.M., Shekara, B.G., Kalyanmurthy, K.N and Shankaralingapa, B.C. 2010. Growth and yield of aerobic rice as influenced by integrated weed management practices. Indian Journal of Weed Science. 42(3):180-183.

Yang, J and Zhang, J. 2010. Crop Management Techniques to enhance harvest index in rice. Journal of Experimental Biology. 61(12):31773189.

Zhao, D.L., Atlin, G.N., Bastiaans, L and Spiertz, J.H.J. 2006. Cultivar weed competitiveness in aerobic rice: Heritability, correlated traits, and the potential for indirect selection in weedfree environment. Crop Science. 46: 372-380.

Zhao, D.L., Bastiaans, L., Atlin, G.N and Spiertz, J.H.J. 2007. Interaction of genotype 9 management on vegetative growth and weed suppression of aerobic rice. Field Crops Research. 100:327340 .

\section{How to cite this article:}

Pavani, R., V. Umamahesh, A.R. Nirmal Kumar and Reddi Ramu, Y. 2018. Influence of Seed Priming Treatments on Biochemical Parameters of Dry Direct Sown Rice (Oryza sativa L.). Int.J.Curr.Microbiol.App.Sci. 7(12): 3066-3078. doi: https://doi.org/10.20546/ijcmas.2018.712.351 